\title{
Research on Measuring Instrument of LoadingCapacity for Insects Sliding on Water Surface
}

\author{
Wang Qingcheng ${ }^{1, \mathrm{a}}$, Yang Xiaodong ${ }^{2, \mathrm{~b}}$ and Mao Ning $^{3, \mathrm{c}}$ \\ ${ }^{1}$ School of Mechanical Engineering, Jilin Teachers' Institute of Engineering \\ and Technology, Changchun 130052, China \\ ${ }^{2}$ School of Mechanical and Electric Engineering, Changchun Institute of \\ Technology, Changchun 130021, China \\ ${ }^{3}$ China North Vehicle Research Institute, Beijing 100072, China

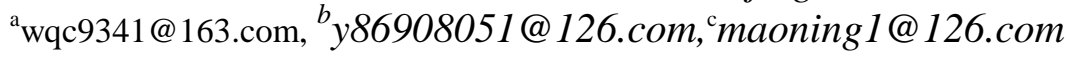

\begin{abstract}
A device of measuring micro-force was designed, which can measure the maximum loadingcapacityof the insect's leg which can slide on water surface. The device consists of two parts of micro-force measurement and feed motion control, micro-force measurement accuracy is $0.1 \mu \mathrm{N}$, micro-analytical balance was chosen to accomplish this function; The smallest linear feed motion speed is $1 \mathrm{~mm} / \mathrm{min}$, AC servo motor was chosen as the actuator, the servo control system consists of the S3C2410 and drike circuit, etc, fuzzy PID control algorithm has been adopted to achievethis function. Micro analytical balance and servo control system communicate with the compater via RS $232 \mathrm{C}$.
\end{abstract}

Keywords: Loading Capacity; Micro-force measurement; Servo control system; Fuzzy PID controller

\section{Introduction}

Water striders, mosquitoes and other insects can slide freely in the water, whose superior loading capacity have drawn the public attention and become one of the hot topics researched by scientists and engmeering technieians in the world [1-3]. Jiang et al., [4] deemed that this is realized by super-hydrophobicity resulting from special micro/nano structure of water strider's leg, and they measured the maximal loadingcapacity of which water strider's single hind leg pierces the water surface, equal to 15 times of its body weight. Wu et al., [5] measured the maxinal loadingcapacity of mosquitoes' single hind leg equal to 23 times of its body weight. But micro force measuring equipment not introduced in detailin paper [4-5], at present, it has been reported that a kind of the instrumentused to measure the loading capacity of the insectss tiding on water surface.

This pape designed a kind of the micro force measuring device, which can measure loading capacity of the insectssliding on water surface.The range of the measurement instrument is $1-5000 \mu \mathrm{N}$, the precision is $0.1 \mu \mathrm{N}$, the feed speed controlling insect downward is $1 \mathrm{~mm} / \mathrm{min}$.

\section{Overall Design}

The overall structure of the micro force measuring device is shown in Figure 1, the micro force measuring element can measure loading capacity value of the insects on water surface, which can communicate with the microcomputer through RS-232C serial port, the measured data is analyzed and processed by upper computer. Servo control system receives the upper 
microcomputer instruction, controlling the insects move downward. Lead screw nut pair can turn rotation movement of the ac servo motor into linear motion in the direction of up and down, nut and slider fixed together with screws, which makes linear movement along the guide rail. Guide rail can reduce the oscillation amplitude of the slider movement process, which can guarantee the stability of the insects moving downward in the test. Scale is made of steel material, which fixed with the slider together with screws. The insect is fixed on the scale with double-sided tape when the experiment is conducted.



Figure 1. Micro Force Nieasuring Device Structure Diagram 3. Micro Force Measuring Element

Micro force measuring element adopted micro analytical balance (A\&D Company, GH 252)its precision is $0.1 \mathrm{mg}$, put the position of balance should be solid, lest cause vibrations. In order to avoid disturbance and affect its precision of the balance, which should stay away from heat sources an conditioning, and the equipment producedmagnetic field. Photos of the balance as shown in Figure 22

Figure 2. Photo of the Microanalytical Balance 
Before using balance, adjust the levelerfirst of all, to keep balance. The main function keys of balance are as follows. ON/OFF - on or off balance, CAL - use the built-in weight calibration balance, RE-ZERO - get rid of tare weight, make balance shows zero.

The balance (GH-252 Type) is communication equipment with DEC data type, which can connect with the microcomputer through RS-232C interface. The balance can transmitted weighing data to the micro computer through WinCT software, which has two kinds of communication methods of Rscom and Rskey. Through Rskey communication method, the weighing data can be continuous or interval data input to the Excel of the computer, then Excel software analysis of weighing data, showed the total number, mean, standard error, the maximum and the minimum, etc through figure and table. Through Rskey communication method, computer can also send instructions to balance, control the operation of the balance.

\section{Control System of Feed Movement}

Over the years, because of the rapid development of microelectronics technology and control theory and technology,control system of AC servo motor has been developing rapidly. Speed control performance of AC servo motor is superior overcome many ísadvantagesof DC servo motor.

\subsection{PMSMMathematical Model}

The measuring device applied of permanent magnet synchronous ac servo motor (PMSM), which has a simple structure, small volume, high efficiency, high power factor, small moment of inertia, strong overload capacity, reliable running etcso PMSM is widely used in the field of automation control. The paper choosesPMSM as executive component, which can converts the electrical signal from upper computer into angular displacement or angular velocity of the motor shaft.

The direction of the fundamental wave magnetic field of permanent magnet is $\mathrm{d}$ axis, and $\mathrm{q}$ axis is electrical angle advancing $90^{\circ}$ along the direction of rotation. Counterclockwise direction is the positive direction, $\mathrm{d}-\mathrm{q}$ axis voltage balance equation is

$$
\left(\begin{array}{l}
u_{d} \\
u_{q}
\end{array}\right)=\left(\begin{array}{cc}
R+p L_{d} & -\omega, L \\
-\omega_{r} L_{d} & B+p L_{q}
\end{array}\right)\left(\begin{array}{l}
i_{d} \\
i_{q}
\end{array}\right)+\left(\begin{array}{l}
0 \\
\omega_{r} \psi_{f}
\end{array}\right)
$$

The magnetic torque equation of PMSM is

$$
T_{e m}=P_{n}\left[\psi_{f}+\left(L_{d}-L_{q}\right) i_{d}\right] i_{q}
$$

Mechanical motion equationof PMSM is

$$
\mathrm{T}_{\mathrm{em}}=T_{L}+B W_{m}+J d \omega_{m} / d t
$$

In the equation (2)(3), $u_{q}, i_{q}$ is voltage and current of the motor shaft, $\mathrm{R}$ is resistance of the stator, $L_{q}$ is inductance of the armature, $\mathrm{P}$ is differential operator, $\omega_{r}$ is the rotor angular frequency, $T_{L}$ is the load torque, $p_{n}$ is motor logarithmic, B is coefficient of friction, $\omega_{m}$ is rotor angular velocity, $\mathbf{J}$ is rotational inertia of the motor, $\psi_{f}$ is permanent magnet of constant flux. 
When applied method of control SPWMcurrent to solve coupling scheme of the approximate linear, the current vector and the rotor magnetic field vector is about $90^{\circ}$. $i_{d}=0$,

$$
\begin{aligned}
& u_{q}=R i_{q}+L_{q} P+\omega_{r} \psi_{f} \\
& u_{d}=\omega_{r} L_{p} p i_{q} \\
& T_{e m}=p_{n} \psi_{f} i_{q}=K_{t} i_{q} \\
& p i_{d}=u_{q} / L_{q}-R_{s} i_{q} / L_{q}-\omega_{r} \psi_{f} / L_{q} \\
& d \omega_{m} / d t=K_{t} i_{q} / J-T_{L} / J-B \omega_{m} / J
\end{aligned}
$$

In the equation (6), $K_{t}$ is the torque coefficient. To make the control goal is mote clear, the equation(7), (8)are transformed into state space form. $i_{q}$ and $\omega_{m}$ was selected as the state variables, get the dynamic mathematical model of PMSM as follows.

$$
\left(\begin{array}{l}
d i_{q} / d t \\
d \omega_{m} / d t
\end{array}\right)=\left(\begin{array}{ll}
-R_{s} / L_{q} & -\psi_{f} / L_{q} \\
K_{t} / J & -B / J
\end{array}\right)\left(\begin{array}{l}
i_{q} \\
\omega_{m}
\end{array}\right)+\left(\begin{array}{l}
1 / L_{q} \\
0
\end{array}\right)\left(u_{q}\right)+\left(\begin{array}{l}
0 \\
-T_{L} / J
\end{array}\right)\left(\omega_{m}\right)
$$

The structure diagram of the motor simplified model is obtained, as shown in Figure 3.

\subsection{Control System Hardware}

Servo motor control gystem is two level of structure, upper computer is a microcomputer, lower computer is servo motor control system, which is made up of Samsung S3C2410 and drive circuit. S3C2410 can complete the corresponding task by receiving the instructions of upper computer, and collected the speed signal in the process, hardware composition of servo control systemas shown in Figure 4.

Mainfunction of servo control system is to control the motor speed. Speed sensor can detect actual the motor speed, and transmits the signalto servo control system, adjust the mocor speed through comparing target speed and actual speed, until output speed can meet the requirements of speed. The magnetic coupling has the ability to suppress noise and prevent the strong electromagnetic interference. 


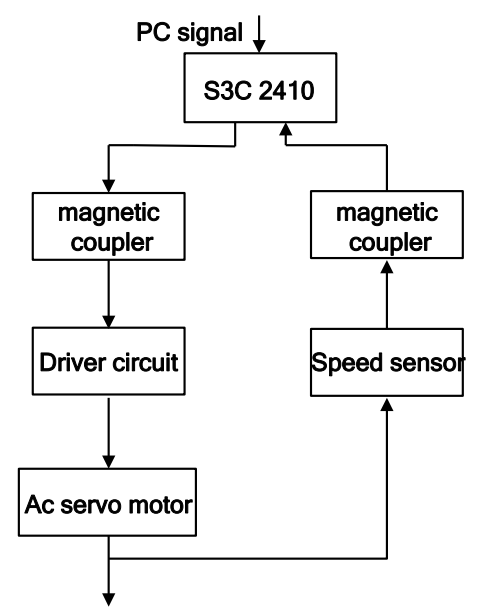

Figure 4. Hardware Structure of Servo Control System

\subsection{Fuzzy PID Controller}

The traditional PID controller is a widely used method its algorithm ideas is

$$
\begin{aligned}
& u(t)=K_{p} e(t)+K_{i} \int_{0}^{t} e(t) d t+K_{d} \frac{d e(t)}{d t}(10) \\
& e(t)=r(t)-c(t)
\end{aligned}
$$

In the equation (10), (11), $e(t)$ is input signal of the controller, $u(t)$ is output signal of the controller, $r(t)$ is target input ofthe controller $c(t)$ is feedback signal of control object, $K_{p}$ isproportionality coefficien of the controller, $K_{i}$ isintegral gain coefficientofthe controller, $K_{d}$ is differential gain coefficient of the controller.

Self-tuning parameters is fixed in the traditional PID, which can not adjust online parameters of $K_{,}, K_{i}$ and $K_{d}$, secontrol effect is not very ideal. Combining fuzzy control and traditional PID control, he paper design a fuzzy controller[6], which can use the current control deviation, considering the change of dynamic characteristics in control process, in view of practical experrence of the specific process, according to the control requirement or objective function, through the fuzzy rules reasoning, realizing self-tuning online PID parameters of $K_{p} \quad K_{i}$ and $K_{d}$. The structure of fuzzy controller as shown in Figure 5.
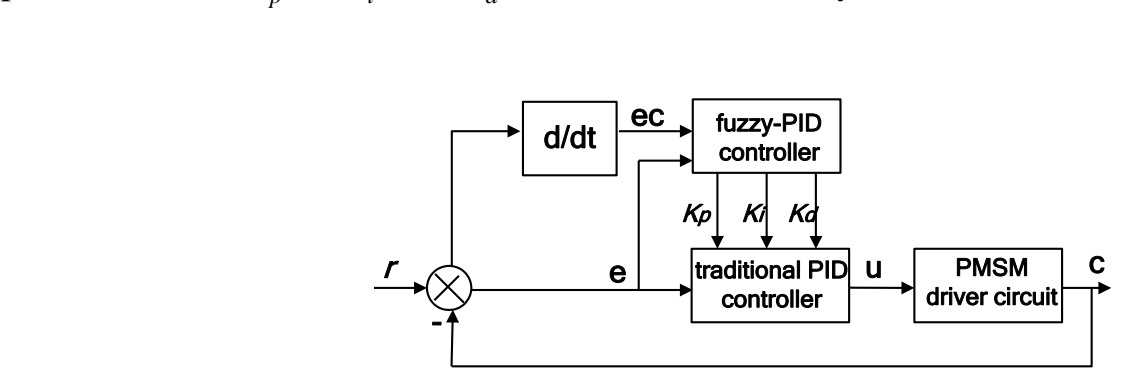

Figure 5. The Structure of Fuzzy-PID Controller 
In the fuzzy PID control system, rotatespeed deviation (e) and change rate deviation (ec)were selected as the input language variable, $K_{p}, K_{i}$ and $K_{d}$ were selected as the output language variable, all each language variables are selected trigonometric function or Gaussian functionas membership function. 7fuzzy subset were taken in changing domain of input and output language variable, which is respectively \{NB NM NS Z PS PM PB $\}$, which can quantize as $\{-3,-2,-1,0,1,2,3\}[7]$.Membership function and changing domain of $K_{p}, \quad K_{i}$ and $K_{d}$ is shown in Figure 6.

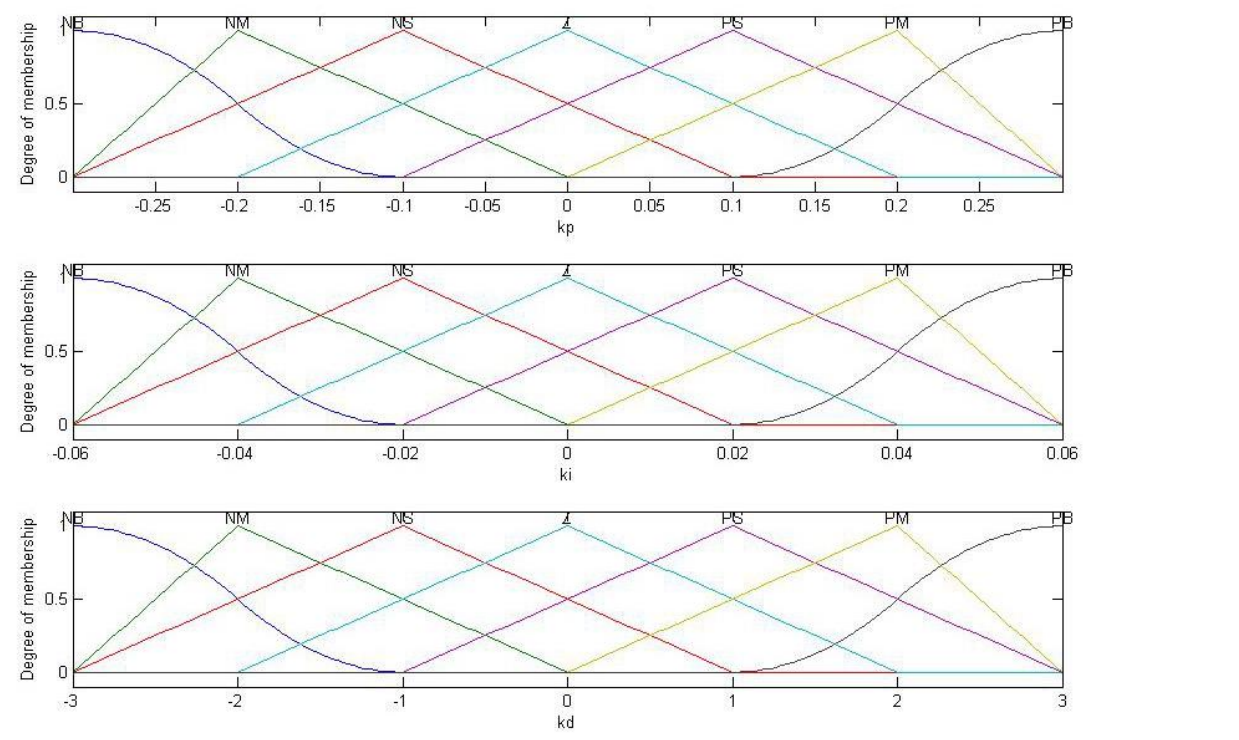

\section{Figure 6. $K_{p}, \quad K_{i}$ and $K_{d}$ Membership Curve}

Fuzzy control rutes is to find out the fuzzy relationship between rotate speed deviation $(e)$, change rate deviation (ec) and three parameters of $\operatorname{PID}\left(K_{p}, K_{i}\right.$ and $K_{d}$ ). Through continuous testingspeed deyjation(e) and change rate deviation $(e c), K_{p}, K_{i}$ and $K_{d}$ are modified online according to the principle of fuzzy control, until the requirements of different e and ec have been meet, so that the controlled object has a good dynamic and static performance[8].Considering the role of $K_{p}, \quad K_{i}$ and $K_{d}$ in different time and the interconnected relationship between each other, PID parameter were have been adjusted According to the engineering design personnel's technical knowledge and practical operation experience, through repeated experiments, obtain fuzzy control rule table of $K_{p}$, $K_{i}$ and $K_{d}$, as shown in Table 1.

Through adopt gravity method, the inverse blur inverse fuzzy processing is conducted, $\Delta K_{p}, \Delta K_{i}$ and $\Delta K_{d}$ were obtained, placed in equation(12), calculation and fix the current $K_{p}, \quad K_{i}$ and $K_{d}$, complete the PID parameter online self-tuning. 
Table 1. $K_{p}, \quad K_{i}$ and $K_{d}$ Fuzzy Control Rules

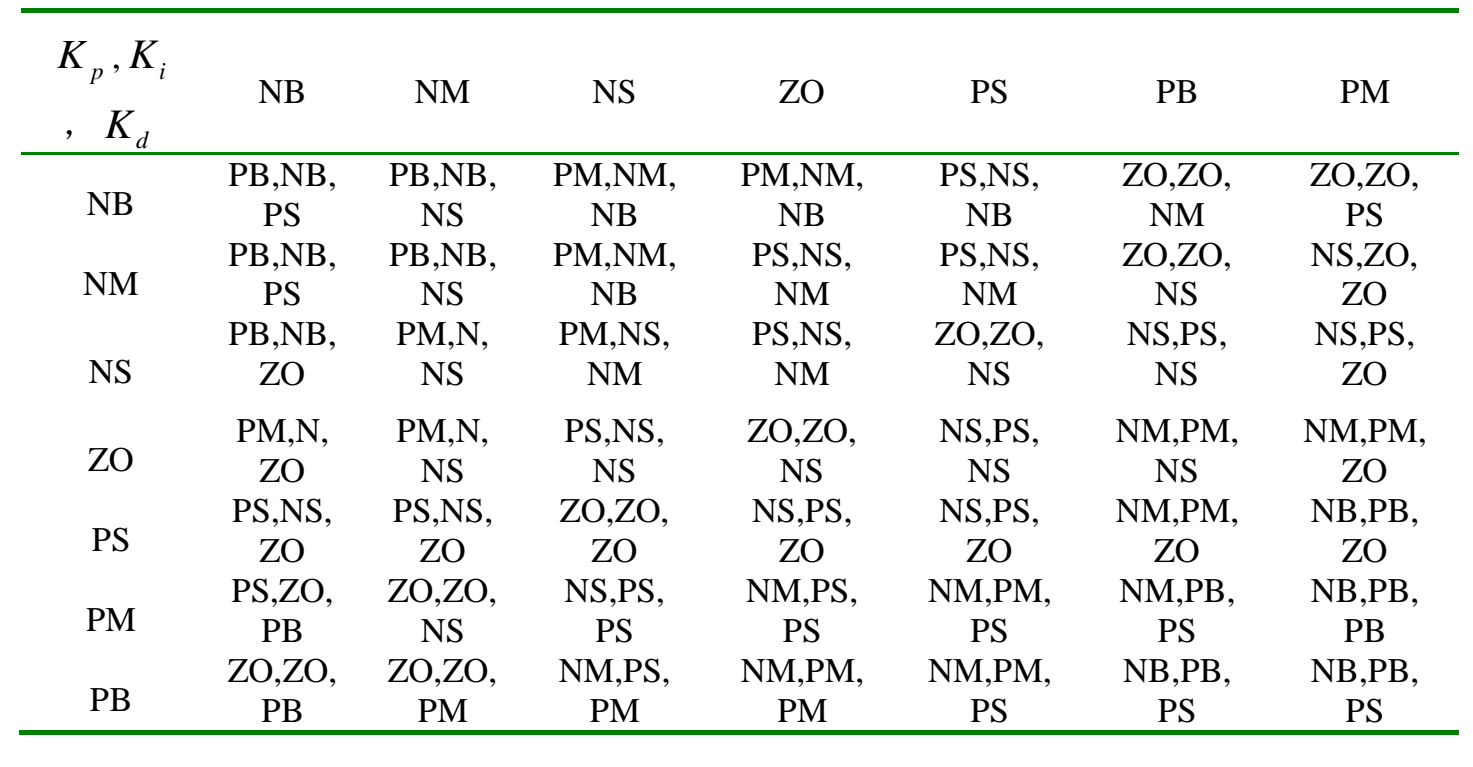

\subsection{Feed Speed Measurement Test}

$$
\left\{\begin{array}{l}
K_{p}=K_{p}^{\prime}+\Delta K_{p} \\
K_{i}=K_{i}^{\prime}+\Delta K_{i} \\
K_{d}=K_{d}^{\prime}+\Delta K_{d}
\end{array}\right.
$$

Using the torque speed Sensor (CX-30) and related equipmentas measuring equipment, which is connected to a computer by RS-232C, the time response of speed have been analyzedquantitatively The experiment ss under the condition of the zero speed, Input is the step function with $500 \mathrm{r} /$ minrotate speed, data collected were made curve, as shown in Figure 7.

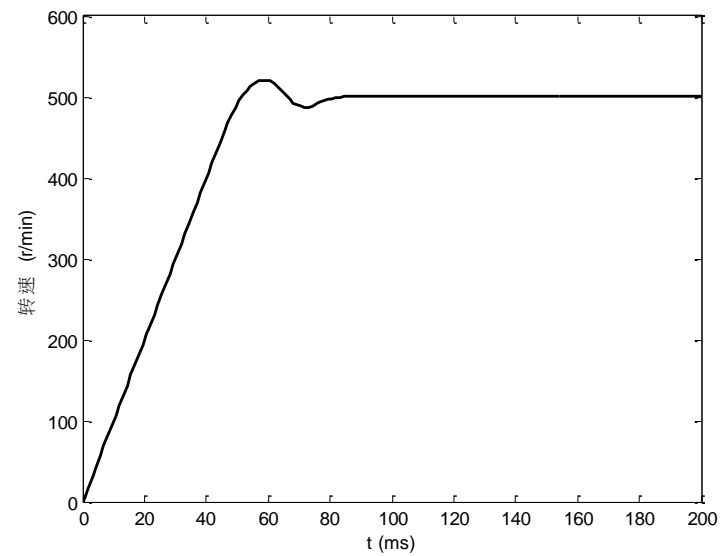

Figure 7. The Time Response of Speed Curve of Rotate Speed 
Seen from Figure 5, response time is less than $80 \mathrm{~ms}$ for $500 \mathrm{r} /$ minrotate speed, response speed of the system is faster. The motor rotate speedpasses through reducer and lead screw nut pair, feed speedis $1 \mathrm{~mm} / \mathrm{min}$.

\section{Conclusion}

(1) This design is based on the actual demand of scientific experiments, which can measure loading capacityof theinsects sliding on water surface.

(2) The range of the force measuring device is $1-5000 \mathrm{uN}$, precision is $0.1 \mathrm{uN}$, the measured data can be analyzed and processed by the upper microcomputer.

(3) Through fuzzy controller,linear feed speed controlling the insects down ward is about 1 $\mathrm{mm} / \mathrm{min}$.

\section{Acknowledgement}

This work was supported by National Natural Science Foundation of China(51275055), Jilin Province Science and Technology Development Project(20140101058JC)and Jilin Provincial Department of Education funded project (2013370).

\section{References}

[1] M. Dickinson, "Animal locomotion: how to walk on water", Nature, vol.424, no.6, (2003), pp. 621622.

[2] D. L. Hu, B. Chan, W. John and M. Bush, "The hydrodynamics of water strider locomotion", Nature, vol.424, no.11, (2003), pp. 663-666.

[3] X. F. Gao and L. Jiang, "Water-repellent legs of water striders", Nature, vol. 432, no. 8, (2004), pp. 26-26.

[4] X. Q. Kong and C. W. Wu, "Superior water repellency of mosquito legs with hierarchical micronano structures", Chinese science bulletin, vol.s5, no.16,(2010), pp.1589-1594.

[5] N. Sun and Y. H. Liang, "Fuzzy PID controller design for A AC permanent magnet synchronous motor servo system”, Machinery Design \& Manufacture, vol.11, no.7, (2009), pp.182-183.

[6] D. L. Gong, Q. Z. Liao, S.M. Wei and L. Guo, "AC Servo Motor Control System Based on ARM7", Development \& Innovation of Machinety \& Electrical Products, vol.23, no.5, (2010), pp. 107-108.

[7] L. Chi, "Ac servo motor of the fuzzy PID control and GUI design", Journal of Qiqihar university, vol.27, no $3,(2003), \mathrm{pp} .21-24$

[8] H. P. Pang, C. J. Liu and S. Y. Jiang, "Sliding mode fuzzy control for permanent magnetic synchronous motor AC seryo systems", Electrical machine and control, vol.10, no.6, (2006), pp.576579.

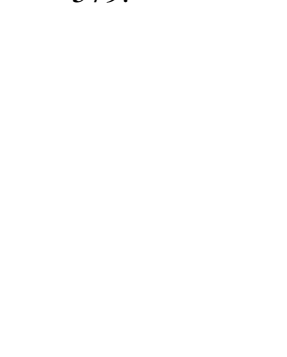

